\title{
Robust synchronization control of multiple vessels with communication
}

\author{
delays \\ Zhou Yuanwei ${ }^{1, a}$, Ma Yanqin ${ }^{2, b}$ \\ ${ }^{1}$ College of Mechanical and Electrical Engineering, Harbin Engineering University, 150001, China \\ ${ }^{2}$ Qingdao Branch, Institute of Acoustics, Chinese Academy of Sciences, 266023, China \\ ${ }^{a}$ E-mail: zhouyuanwei@126.com, ${ }^{b}$ E-mail: mayanqin@mail.ioa.ac.cn
}

KeyWords: robust control, synchronization control method, undirected communication topology, communication delays

Abstract: With the communication delays in the communication topology, the synchronization motion of multiple marine vessels is discussed. A novel synchronization control method that is based on distributed control strategy and graph theory is presented. The synchronization error and the trajectory tracking error of each vessel are introduced to the controller. Besides, an undirected communication topology is designed to describe the communication relations among vessels. To validate the designed controller is robust to the communication delays, constant time delays in the communication network are introduced. Finally, four marine vessels with communication delays are simulated by computer simulation tests. It is shown that the proposed control strategy can solve the synchronization of multiple vessels well.

\section{Introduction}

With the increasing complexity of marine missions, multiple marine vessels systems can perform tasks more efficiently than a single vehicle or can accomplish tasks not executable by a single one, it can be considered as a concept for the emergence of new capabilities [1,2].

Recently, synchronization control of multiple agents has been extensively studied in different fields. And as the development of synchronization control of multiple agents, there are several synchronization control approaches, which are proposed. For example, distributed cooperative attitude synchronization control approach has been discussed in [3]. And adaptive control is a typical scheme used to address the synchronization motion [4, 5]. Besides, using cascaded system theory and graph theory, a distributed attitude cooperative control strategy is studied in [6]. And based on graph theory, two synchronization controllers in cooperative and coordinated schemes were designed [7]. And for coordinated synchronization control of multiple marine vessels, E. Kyrkjebø introduced a virtual structure into the leader-follower coordinated control scheme[8]. And a cooperative synchronization control law of multiple autonomous underwater vehicles is presented by C. R. Xin[9], in both state feedback and output feedback are designed to achieve synchronization. And without the mathematical model of the leader, E. Kyrkjebø designs a nonlinear observer to estimate the unknown states of the follower and a feedback controller was designed $[9,10]$. The synchronization of multiple systems with time delays is discussed in $[11,12]$.

In this paper, the synchronization movement control of multiple vessels is discussed. A weighted undirected graph is introduced to describe the information exchange among vessels, then the available synchronization error among vessels is taken into controller, at the same time, when the time delays are considered, the controller demonstrates robustness to constant time delays. 


\section{Preliminary Knowledge and Vessel Model}

Graph Theory Let $G=\{\mathrm{V}, \mathrm{E}\}$ describe the information exchanges among vessels. A node in the graph represents a vessel. The edges represent the information exchange links among the vessels. Node $j$ is a neighbor of node $i$ if $\left(v_{i}, v_{j}\right) \in \mathrm{E}(\mathrm{G})$. Assume matrix $A$ is the adjacency matrix of graph $G$, and $a_{i . j} \in A$ is defined as $a_{i, j}>0$, if $\left(\mathrm{v}_{i}, \mathrm{v}_{j}\right) \in E, i \neq j, \quad a_{i, j}=0$, if $\left(\mathrm{v}_{i}, \mathrm{v}_{j}\right) \notin E$.For all $\left(v_{i}, v_{j}\right) \in \mathrm{E}(\mathrm{G})$, If the $\left(v_{j}, v_{i}\right) \in \mathrm{E}(\mathrm{G})$, then the graph is undirected.

Mathematical Model of the Vessel The 3 DOF surface motion model of the vessel can be described as:

$$
\eta \ll=R(\psi) v \quad M \&+D(v) v+C(v) v=\tau_{v} .
$$

where $\eta=[n, e, \psi]^{T}$ is the position and heading in the earth-fixed reference frame, and $v=[u, v, r]^{T}$ is the surge, sway velocity and yaw angular velocity with regard to the body-fixed reference frame.

The motion mathematical model of the vessel in the earth-fixed reference frame can be written as:

$$
\nu=R^{T}(\psi) \eta \alpha \quad M(\eta) \alpha+D(\eta, \eta \sin \alpha+C(\eta, \eta \sin \alpha=\tau(\eta) .
$$

\section{Synchronization Controller Design}

Synchronization Controller Design with Communication Delays When the communication network is unreliable, it is desired to take time delays into account. In the subsequent analysis, it is assumed that $T_{\mathrm{ij}}$ denotes the constant time delay, and $T_{\mathrm{ij}}=T_{\mathrm{ji}}=T$. And in this time delayed network, a classical communication delayed model of multi-agent is adopted.

Firstly, the position trajectory error of the $i-t h$ vessel is defined

$$
e_{1 i}=\eta_{i}(t)-\eta_{d i}(t)+\int_{0}^{t} \Gamma_{i}\left(\eta_{i}(\lambda)-\eta_{d i}(\lambda)\right) d \lambda
$$

where $\Gamma_{i}$ is a positive diagonal matrix to be designed.

There are different ways to regulate the synchronization error, and the synchronization error of the $i-t h$ vessel is adopted as follows

$$
\left.e_{2 i}(t)=\sum_{i \neq j} K_{i j}\left(\eta_{i}\left(t-T_{i j}\right)-\eta_{d i}\left(t-T_{i j}\right)\right)-\left(\eta_{j}\left(t-T_{j i}\right)-\eta_{d j}\left(t-T_{j i}\right)\right)\right)=\sum_{i \neq j} K_{i j}\left(\eta_{i}\left(t-T_{i j}\right)-\eta_{j}\left(t-T_{j i}\right)-d_{i j}\left(t-T_{j i}\right)\right) .
$$

where $K_{i j}$ is a positive diagonal matrix $K_{i j}=\operatorname{diag}\left\{a_{i j}\right\}$. And $a_{i j}$ is the element of the weighted adjacency matrix A. $d_{i j}$ is defined as:

$$
d_{i j}=\eta_{d i}-\eta_{d j} .
$$

Then the global error of the $i-t h$ vessel can be written as:

$$
e_{i}=e_{1 i}+\int_{0}^{t} e_{2 i}(\omega) d \omega \text {. }
$$

The control law $\tau$ for the $i-t h$ vessel is with time delays denoted as: 


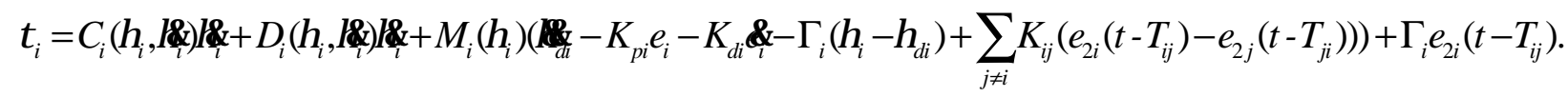

Therefore, the error of the whole system can be calculated combing (3) and (7) as follows.

$-K_{P} e-K_{d} \& K_{a} \&(t-T)$.

where $K_{p}=\operatorname{diag}\left\{K_{p i}\right\}, \quad K_{d}=\operatorname{diag}\left\{K_{d i}\right\}, K_{a}=L \otimes I_{3 \times 3}$.

By the Leibnitz formula, the error can be written as:

$$
\left.\&(t-T)=\int_{t-T}^{t} \& w\right) d w \text {. }
$$

Combine (2) and (9), which leads to

$$
\left.-K_{p} e-K_{d} \& K_{a}\left(\&-\int_{t-T}^{t} w\right) d w\right) \text {. }
$$

Define $\delta=\left[e^{T}, \&\right]^{T}$, therefore (10)yields:

$$
\delta=A \delta-B \int_{t-T}^{t} \delta\left({ }_{w}\right) d w .
$$

$$
\text { where } A=\left[\begin{array}{cc}
0 & I \\
-K_{p} & -K_{d}+K_{a}
\end{array}\right], B=\left[\begin{array}{cc}
0 & 0 \\
0 & K_{a}
\end{array}\right] \text {. }
$$

Proof: Considering the positive definite candidate Lyapunov function for the delayed system as:

$$
V=\delta^{T} H \delta+\int_{t-T}^{t} \delta^{T}(w) P \delta(w) d w .
$$

where $H, P$ are positive definite matrix. Then the derivative of the Lyapunov function yields

$$
\begin{aligned}
\mathscr{\alpha} & =\delta^{T} H\left(A \delta-B \int_{t-T}^{t} \delta\left({ }_{w}\right) d w\right)+\delta^{T} P \delta-\delta^{T}(\mathrm{t}-T) P \delta(\mathrm{t}-T) \\
& \leq \delta^{T}(H A+P) \delta+\delta^{T} H B \delta(\mathrm{t}-T)-\delta^{T}(\mathrm{t}-T) P \delta(\mathrm{t}-T)-\delta^{T} H B \delta \\
& \leq-\delta^{T}\left(H B-H A-P-\frac{H B}{4 \varepsilon_{2}}\right) \delta-\delta^{T}(\mathrm{t}-T)\left(P-\varepsilon_{2}\right) \delta(\mathrm{t}-T) .
\end{aligned}
$$

By adjusting $A, B, H, P, \varepsilon_{2}$ to insure that $H B-H A-P-H B / 4 \varepsilon_{2}>0$ and $P-\varepsilon_{2}>0$. So that $\Leftrightarrow \leq 0$.

Hence, all errors in the whole system are bounded, and it can be shown $\lim _{t \rightarrow \infty} \delta=0, \lim _{t \rightarrow \infty} \delta(t-T)=0$, then $\lim _{t \rightarrow \infty} e=0, \lim _{t \rightarrow \infty} \&=0$, and combine (15),(19) with(6), it can be seen that the position converges to synchronization, which means $\lim _{t \rightarrow \infty} \eta_{i}-\eta_{j}=d_{i j}, \lim _{t \rightarrow \infty} \eta_{i}=\eta_{d i}$, and the

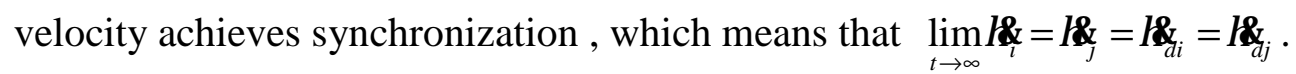

\section{Simulations}

To validate the effectiveness of the proposed synchronization control law, some simulations are carried out, the mathematical model of vessels are the CyberShipII.

The directed communication topology of vessels is shown in Fig.1. And the weighted adjacency 
matrix of the communication graph is $A=0.5 \times\left[\begin{array}{llll}0 & 1 & 1 & 1 \\ 1 & 0 & 0 & 0 \\ 1 & 0 & 0 & 1 \\ 1 & 1 & 1 & 0\end{array}\right]$. The communication delays are $\begin{array}{lllll}\text { chosen } & T=1 s & \text { The } & \text { controller }\end{array}$ are $K_{p i}=0.5 \times \operatorname{diag}\{1,1,1\}, K_{d i}=\operatorname{diag}\{15,15,18\}, K_{i}=0.5 \times \operatorname{diag}\{1,1,1\}, \Gamma_{i}=0.8 \times \operatorname{diag}\{1,1,1\}$.

The simulation results are shown in Fig. 2-Fig. 7 The synchronization error of lateral velocity Fig. 8. And the meaning of vessel ${ }_{i j}$ is the vessel $i$ and vessel $j$. The trajectories of four vessels in the North-East coordinate as shown in Fig. 2, the synchronization error are shown in Fig. 3-Fig. 7 The synchronization error of lateral velocity Fig. 8. From the movement of the vessels, it can be seen with the designed controller, the four vessels can follow the desired trajectory of tracking while maintaining the synchronization between the adjacent vessels, which can perform collaborative tasks at sea.

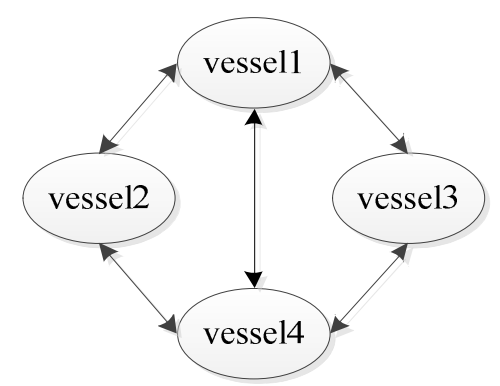

Fig. 1 The communication topology of vessels

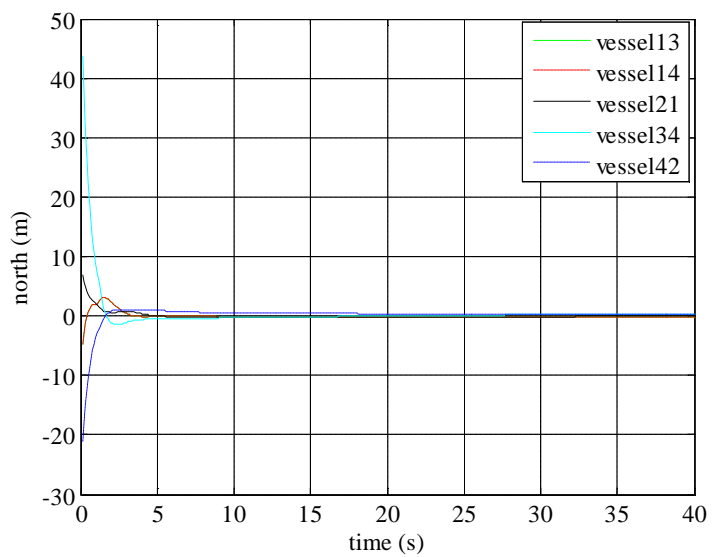

Fig. 3 The synchronization error in north

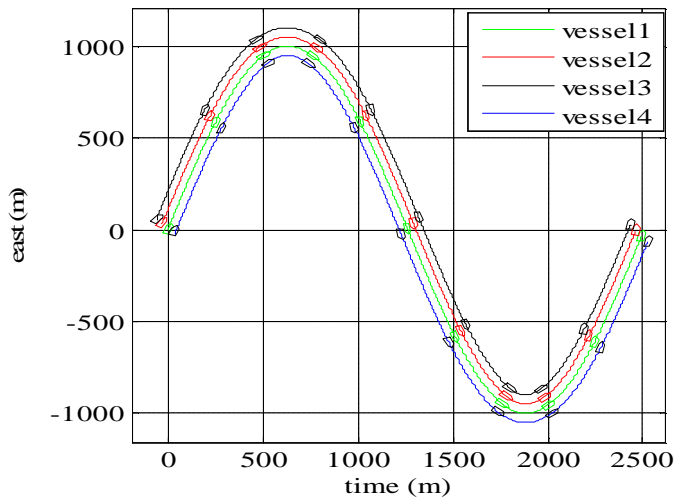

Fig. 2 The movement curve of each vessel

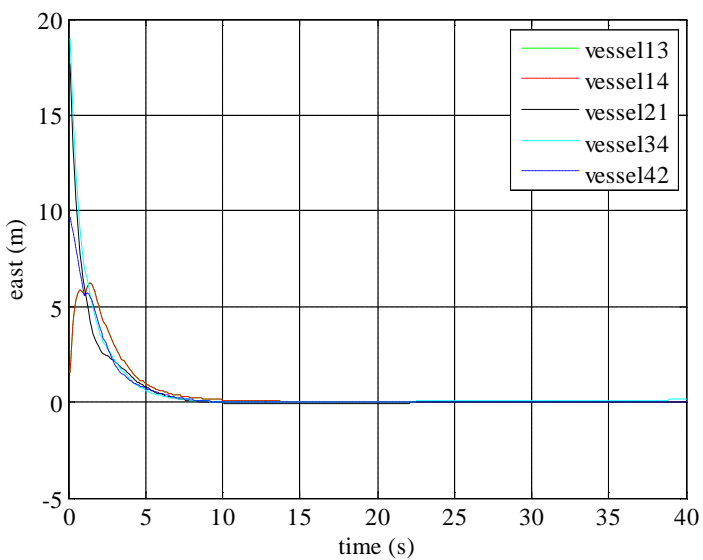

Fig. 4 The synchronization error in the east 

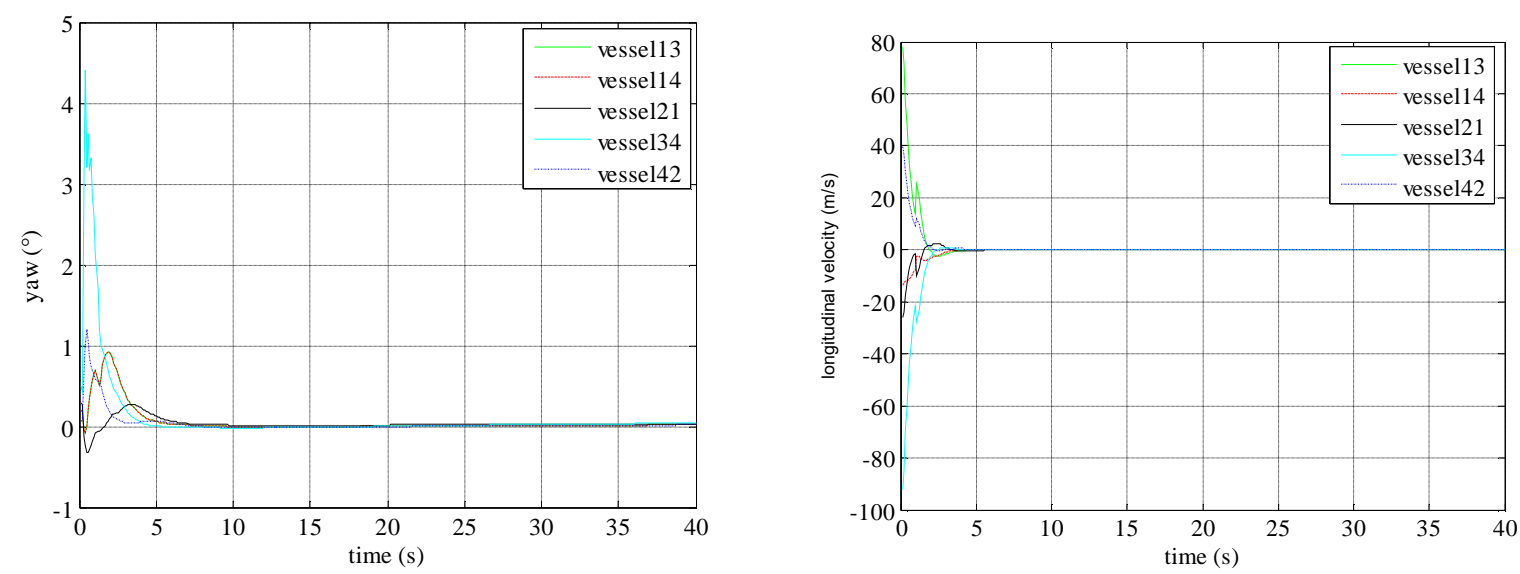

Fig. 5 The synchronization error of yaw Fig. 6 The synchronization error of longitudinal velocity
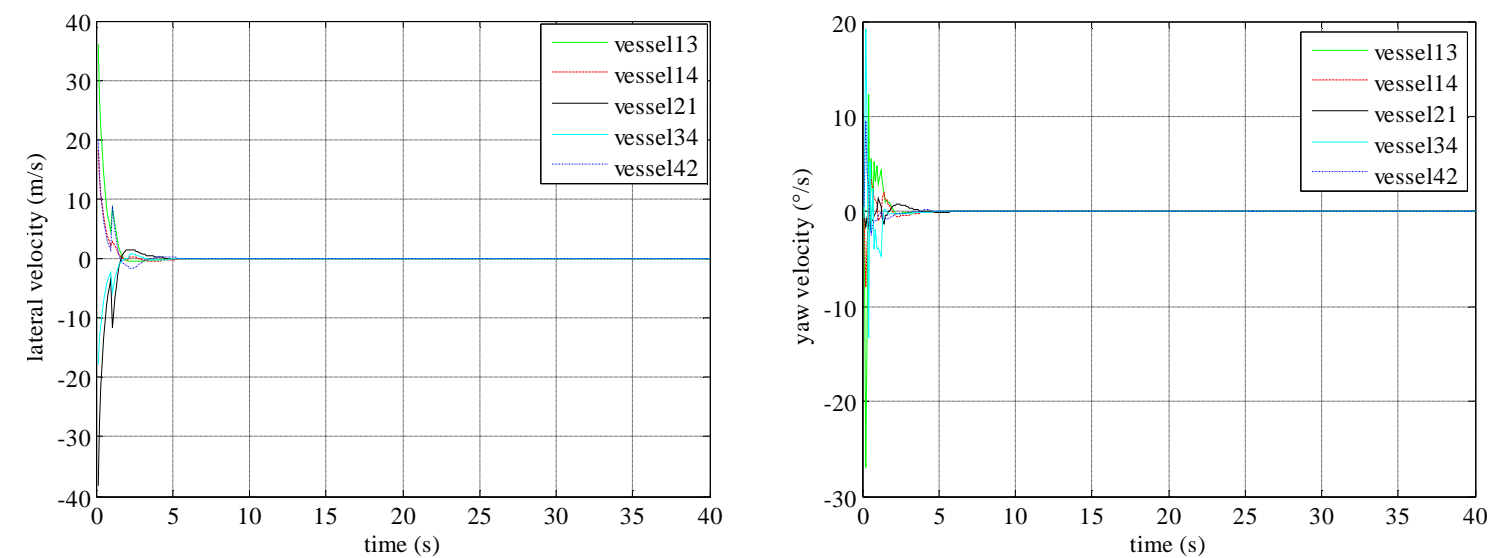

Fig. 7 The synchronization error of lateral velocity

Fig. 8 The synchronization error of yaw velocity

From the synchronization errors of vessels, it can be seen that when considering communication delays, the synchronization errors between vessels eventually tends to zero. And the designed synchronization motion controller is robust to constant communication delays.

\section{Conclusions}

In this paper, a synchronization control approach for controlling vessels is presented. The controller can be seen a nonlinear PD controller. And based on the graph theory, the synchronization errors among vessels are added to the controller. From the simulations, it can be seen that the whole error of the closed-loop system is ultimately bounded. Therefore, the proposed control scheme can achieve synchronization of vessels. In the future work, it is expected that only position and yaw angle of the vessels are available, it may be potential to apply the synchronization control law to non-holonomic system.

\section{Reference}

[1] Murray R M. Recent research in cooperative control of multi-vehicle systems[J]. Journal of Dynamic Systems, Measurement, and Control. 2007.129(5): 571-583.

[2] Fossen T I. Handbook of Marine Craft Hydrodynamics and Motion Control[M]. New York: Wiley-Sons Ltd, 2011.

[3] W. Ren. Distributed cooperative attitude synchronization and tracking for multiple rigid bodies[J]. IEEE Transactions on Control Systems Technology, 2010.18(2):383-392. 
[4] H. Wang. Passivity based synchronization for networked robotic systems with uncertain kinematics and dynamics[J]. Automatica. 2013.49(3):755-761.

[5] D. Sun. Position synchronization of multiple motion axes with adaptive coupling control[J]. Automatica. 2003.39 (6):997-1005.

[6] H. Du and S. Li. Attitude synchronization control for a group of flexible spacecraft[J]. Automatica. 2014. 50(2):646-651.

[7] Y. Bouteraa, 1. Ghommam, N. Derbel et al. Mutual and external synchronization control of multi-robot systems[J]. International Multi-conference on Systems Signals \& Devices. 2010, 28(5):808-813.

[8] E. Kyrkjebø, E. Panteley, A. Chaillet et al. A virtual vehicle approach to underway replenishment [J]. Springer Berlin Heidelberg, 2006, 336:171-189.

[9] C. R. Xin and Y. W. Sheng. Synchronization of multiple autonomous underwater vehicles without velocity measurements[J]. Science China Information Sciences. 2012, 55(7):1693-1703.

[10]E. Kyrkjebø and K. Y. Pettersen. Leader-Follower output reference state feedback synchronization control of Euler-Lagrange systems[J]. Conference on Control \& Automation, 2007:1-6

[11]Y. Bouteraa, J. Ghommam, G. Poisson et al. Distributed synchronization control to trajectory tracking of multiple robot manipulators[J]. Hindawi Publishing Corporation Journal of Robotics, 2011.1-10.

[12]L. Y. Chen and N. Chopra. Controlled synchronization of heterogeneous robotic manipulators in the task space[J]. IEEE transactions on robotics, 2012. 28(1):268-275 